\title{
Resistant hypertension: Renal denervation or pharmacovigilance? Insights from a renal denervation screening program
}

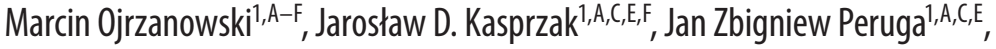 \\ Małgorzata Kurpesa ${ }^{1, B, E}$, Łukasz Jankowski ${ }^{1, B-D}$, Sonu Sahni ${ }^{2,3, C-E}$, Michał Plewka ${ }^{1, A-F}$ \\ ${ }^{1}$ Chair and Clinic of Cardiology, Medical University of Lodz, Poland \\ 2 Department of Primary Care, Touro College of Osteopathic Medicine, New York, USA \\ ${ }^{3}$ Department of Internal Medicine, Brookdale University Hospital Medical Center, New York, USA \\ A - research concept and design; $B$ - collection and/or assembly of data; $C$ - data analysis and interpretation; \\ $D$ - writing the article; $E$ - critical revision of the article; $F$ - final approval of the article
}

Address for correspondence

Marcin 0jrzanowski

E-mail: ojrzan@02.pl

Funding sources

Medical University of Lodz, Poland

Conflict of interest

None declared

Received on November 10, 2017

Reviewed on June 1, 2018

Accepted on February 18, 2019

Published online on November 6, 2019

Cite as

Ojrzanowski M, Kasprzak JD, Peruga JZ, et al. Resistant hypertension: Renal denervation or pharmacovigilance? Insights from a Renal Denervation Screening Program. Adv Clin Exp Med. 2019;28(11):1525-1530.

doi:10.17219/acem/104550

D0I

10.17219/acem/104550

Copyright

Copyright by Author(s)

This is an article distributed under the terms of the

Creative Commons Attribution Non-Commercial License

(http://creativecommons.org/licenses/by-nc-nd/4.0/)

\begin{abstract}
Background. With emerging new therapeutic concepts including renal denervation (RDN), there is a renewed interest in resistant hypertension (ResH). Among patients suspected of having ResH, a definitive diagnosis needs to be established.
\end{abstract}

Objectives. This study presents observations from a standardized single-center screening program for RDN candidates, including medical therapy modification and reassessment.

Material and methods. All patients referred to our center for RDN underwent a standardized screening protocol. Candidates were recruited from among patients receiving no less than 3 antihypertensive drugs, including diuretics with office blood pressure (BP) $>140 / 90 \mathrm{~mm} \mathrm{Hg}$. The assessment included 2 measurements of BP and ambulatory BP monitoring (ABPM). If needed, pharmacotherapy was intensified and the diagnosis of ResH was reconfirmed after 6 weeks. If ResH was persistent, patients were hospitalized with repeated ABPM on day 4. Further, renal CT-angio was performed and a multidisciplinary team discussed the patients' suitability for RDN.

Results. A total of 87 patients with a ResH diagnosis were referred for RDN. Mean office BP was 159/92 $( \pm 7.0 / 6.5) \mathrm{mm} \mathrm{Hg}$ and mean ABPM was $154 / 90( \pm 9.0 / 4.8) \mathrm{mm} \mathrm{Hg}$. The initial medication included angiotensin convertase inhibitors (ACE-I, 78\%), angiotensin receptor blockers (12\%), $\beta$-blockers (85\%), calcium channel blockers (36\%), and diuretics (93\%). During the 18 months of the RDN program, 5 patients underwent RDN and 2 further had ineligible renal anatomy. A new diagnosis of secondary hypertension was made in 21 patients. However, in 59 patients, BP control was achieved after optimization of medical therapy, with a mean ABPM of $124 / 74 \mathrm{~mm} \mathrm{Hg}$. The final treatment included ACE-I (100\%), $\beta$-blockers (92\%), indapamide (94\%), amlodipine (72\%), and spironolactone (61\%). Medication in most of these patients (88\%) included single-pill triple combination (52.5\%) or double combination (35.6\%).

Conclusions. Patients with elevated BP screened for RDN require a rigorous diagnostic workup. Up to 2/3 of patients can be managed with strict pharmacotherapy compliance and pharmaceutical intensification, including single-pill combinations and improved drug compliance. Hasty use of RDN may be a result of poor drug optimization and/or compliance. It does remain a viable treatment option in thoroughly vetted ResH patients.

Key words: compliance, arterial hypertension, resistant hypertension, renal artery denervation 
Arterial hypertension (HA) remains a major public health concern with substantial morbidity and mortality, affecting nearly $25 \%$ of all adults in the industrialized world. More specifically, the national health registry data in Poland suggest that approx. 10.5 million people suffer from HA, accounting for approx. $32 \%$ of the adult population. ${ }^{1}$ Over the past decades, a great deal of research and literature has focused on HA leading to a general consensus about the pathomechanisms, pharmacotherapy and other treatment modalities; however, there remains a subset of patients that do not benefit from the standard treatment algorithm. These patients are thought to suffer from resistant hypertension ( $\operatorname{Res} H)$, which is defined as blood pressure (BP) that remains above the goal in spite of optimal doses of 3 antihypertensive agents of different classes, ideally including a diuretic. ${ }^{2}$ However, in situations where elevated office BP is due to white-coat hypertension, improper BP measurement or medication non-compliance, patients are considered not to have true $\operatorname{ResH} \mathrm{H}$, but rather so-called pseudo-ResH. ${ }^{3}$

In the case of true $\mathrm{ResH}$, a failure of pharmacotherapy leads to more invasive methods of treatment, which are based conceptually on the role of the autonomic nervous system in the pathogenesis of HA. Initially, invasive techniques involved surgical sympathectomy of abdominal organs, ${ }^{4}$ which is usually successful in anti-hypertensive effects but often results in unbearable gastrointestinal distress. Thus, a consensus was reached that renal artery denervation (RDN), a more selective procedure, may be an option for patients with ResH. The authors of the largest clinical trials examining RDN - HTN $1,{ }^{5} \mathrm{HTN} 2{ }^{6}$ and $\mathrm{HTN}^{7}$ - established strict inclusion criteria and procedural guidelines for the consideration of RDN. According to the practice-based guidelines, patients suspected of ResH undergo serial consultations along with confirmatory testing. Due to the complicated screening and verification of clinical suspicions of RDN, there needs to be a consensus on the diagnosis and final qualification for RDN therapy. In this paper, the authors seek to analyze the causes of disqualifications from RDN in patients with a suspected diagnosis of $\mathrm{ResH}$.
Society of Cardiology. ${ }^{2}$ Participation in the study required informed consent, which outlined all the study procedures and potential side effects. The study protocol was broken down into multiple phases: a preliminary phase to confirm the diagnosis of true $\mathrm{ResH}$ and a confirmation/hospital phase. The preliminary phase included screening for true $\mathrm{ResH}$, composed of a detailed medical history and HA analysis, outlined in Fig. 1.

Preliminary phase observations helped to exclude patients with a diagnosis of secondary HA or those with an increased vascular risk (i.e., abdominal aortic aneurysm or atherosclerosis obliterans) in invasive RDN. The patient interview was focused on a detailed history of the patient's HA and past medical and pharmacotherapy. A small subset of patients was found to be mismanaged according to treatment guidelines and a definitive diagnosis of ResH could not be made. In these cases, the pharmacotherapy was optimized and the preliminary stage of the study was repeated after 6 weeks to assess for true ResH. When a diagnosis of $\mathrm{ResH}$ was confirmed during the initial or reassessment visit (after pharmacotherapy optimization), a patient was enrolled in the study and admitted to the hospital.

The second phase of the study, considered the confirmation/hospital stage, is outlined in Fig. 2. A continuation of pharmacotherapy along with routine BP monitoring was followed by blood tests to further exclude any other causes of HA or risks in undergoing RDN.

After all inclusion criteria were met, the patients were screened and qualified to undergo the RDN procedure. $\mathrm{The}_{\text {Symplicity }}^{\mathrm{TM}}$ renal denervation system (Medtronic, Dublin, Ireland) was used to carry out the RDN procedure. Each RDN procedure was conducted by a properly trained operator - a cardiologist, experienced in percutaneous coronary angioplasty procedures and supported by a highly qualified licensed technician sent by the manufacturer. Post-procedure hospitalization lasted an average of 3 days. Ambulatory BP monitoring (ABPM) and a blood test were conducted on the last day of hospitalization just before discharge, and again at 6 months ( \pm 2 weeks) and 12 months ( \pm 4 weeks). The approval of the Medical University of Lodz Bioethics Committee/Institutional Review Board was obtained for this study.

\section{Material and methods}

We conducted a prospective study, enrolling 87 consecutive patients with a preliminary diagnosis of $\mathrm{ResH}$ who were hospitalized in either the cardiology or nephrology wards at the Medical University of Lodz, Poland. For the purposes of the study, ResH was defined as BP that remained above the goal in spite of optimal doses of 3 antihypertensive agents of different classes, 1 ideally being a diuretic, in accordance with the most updated guidelines set forth by the European Society of Hypertension and the European

\section{2 independent office measurements of arterial blood pressure}

- positive qualification when systolic BP was $\geq 140 \mathrm{~mm} \mathrm{Hg}$ or the diastolic BP was $\geq 90 \mathrm{~mm} \mathrm{Hg}$

calculation of estimated glomerular filtration rate (eGFR)

- eGFR score $>30 \mathrm{~mL} / \mathrm{min} / 1.73 \mathrm{~m}^{2}$ was an inclusion criterion

$24 \mathrm{~h}$ blood pressure monitoring (ABPM)

- positive qualification to screening for RDN when mean systolic BP was $>135$ and/or mean diastolic BP was $>85 \mathrm{~mm} \mathrm{Hg}$

Fig. 1. Preliminary phase testing 
continuation of pharmacotherapy

double BP measurement bedside, repeated 3 times a day

blood sampling: morphology, metabolic panel, liver function

computer tomography angiography of abdominal aorta and renal arteries

- in the absence of anatomic aberrations that could interrupt RDN

consultation with endocrinologists and hypertensiologists

\section{exclusion of primary hyperaldosteronism}

- assessment of electrolytes in blood and urine,

- primary aldosterone concentration measurement,

- aldosterone concentration measurement after captopril inhibition test

Fig. 2. Hospital procedures of study protocol

\section{Statistical analysis}

The quantitative data was compared to a standardized bell curve with the Kolmogorov-Smirnov test (Lilliefors modification). When the data was compatible to normal distribution, a mean and standard deviation were used (mean \pm SD). A variance analysis for dependent samples was performed. The calculations were carried out on MedCalc Statistical Software v. 17.11.5 (MedCalc Software bvba, Ostend, Belgium).

\section{Results}

A total of 87 patients (33 female, 54 male) were enrolled in the study. The demographics are shown in Table 1. The mean office BP measurement in the study group was $159 / 92( \pm 8.7 / 6.5) \mathrm{mm} \mathrm{Hg}$ and the mean ABPM measurement was $154 / 90( \pm 9 / 4.8) \mathrm{mm} \mathrm{Hg}$. During the 18 months of enrollment, only 5 patients fully satisfied the inclusion criteria and were qualified to undergo RDN. The primary choice for vascular access was the right femoral artery, and no local complications were noted after the procedure.

All the patients who underwent the RDN procedure were followed up at 6 and 12 months, in accordance with the protocol. A modification of pharmacotherapy was required in 1 patient - a reduction of $\beta$-adrenolytic due to asymptomatic bradycardia. The treatment of other patients was not altered during the observation time. Office BP and ABPM measurements revealed a reduction of overall BP as compared to the initial measurements at the time of enrollment. Blood pressure measurements from consecutive visits are shown in Table 2.

The patients that underwent RDN all had satisfactory primary renal function with estimated glomerular filtration rates (eGFR) $>60 \mathrm{~mL} / \mathrm{min} / 1.73 \mathrm{~m}^{2}$. There was no significant deterioration of renal function

Table 1. Demographic and clinical characteristics of the study population

\begin{tabular}{|l|c|}
\hline \multicolumn{1}{|c|}{ Parameter } & Mean \pm SD \\
\hline Age [years] & $63.58 \pm 10.01$ \\
\hline Gender (female/male) & $33(37.9 \%) / 54(62.1 \%)$ \\
\hline Diabetes mellitus type 2 & $22(25.3 \%)$ \\
\hline Coronary artery disease & $24(27.6 \%)$ \\
\hline Hypercholesterolemia & $23(26.4 \%)$ \\
\hline Atrial fibrillation & $11(12.6 \%)$ \\
\hline History of stroke & $8(9.2 \%)$ \\
\hline eGFR (MDRD) $\left[\mathrm{mL} / \mathrm{min} / 1.73 \mathrm{~m}^{2}\right]$ & $73.67 \pm 23.11$ \\
\hline Creatinine $[\mathrm{mg} / \mathrm{dL}]$ & $1.06 \pm 0.29$ \\
\hline Time of HA therapy [years] & $15.01 \pm 5.99$ \\
\hline
\end{tabular}

SD - standard deviation; eGFR - estimated glomerular filtration rate; MDRD - eGFR estimation formula; HA - arterial hypertension.

Table 2. Office BP measurements and ABPM measurements during the follow-up period in patients that underwent RDN

\begin{tabular}{|c|c|c|c|c|}
\hline Parameter & $\begin{array}{c}\text { M0 BP } \\
(\text { mean } \pm S D)\end{array}$ & $\begin{array}{c}\text { M6 BP } \\
(\text { mean } \pm S D)\end{array}$ & $\begin{array}{c}\text { M12 BP } \\
\text { (mean } \pm \mathrm{SD})\end{array}$ & p-value \\
\hline \multicolumn{5}{|c|}{ Mean office BP measurements } \\
\hline Systolic BP & $158.80 \pm 6.40$ & $134.70 \pm 4.40$ & $130.50 \pm 1.32$ & $<0.001$ \\
\hline Diastolic BP & $88.30 \pm 4.56$ & $85.10 \pm 4.55$ & $81.60 \pm 6.19$ & 0.14 \\
\hline \multicolumn{5}{|c|}{ ABPM results } \\
\hline 24-hour systolic BP & $141.40 \pm 4.83$ & $127.60 \pm 8.38$ & $130.60 \pm 6.39$ & 0.015 \\
\hline 24-hour diastolic BP & $82.60 \pm 4.34$ & $70.80 \pm 7.82$ & $71.20 \pm 6.30$ & 0.026 \\
\hline Systolic BP - day & $145.00 \pm 5.24$ & $132.20 \pm 11.67$ & $135.40 \pm 6.19$ & 0.017 \\
\hline Diastolic BP - day & $86.20 \pm 5.26$ & $75.80 \pm 10.71$ & $75.60 \pm 7.50$ & 0.053 \\
\hline Systolic BP - night & $132.00 \pm 4.36$ & $120.20 \pm 5.50$ & $123.00 \pm 4.18$ & 0.049 \\
\hline Diastolic BP - night & $75.80 \pm 6.91$ & $61.60 \pm 7.86$ & $65.40 \pm 5.90$ & 0.088 \\
\hline
\end{tabular}

BP - blood pressure; M0, M6, M12 - consecutive follow-up visits at the end of hospitalization, after 6 and 12 months post hospitalization; SD - standard deviation; ABPM - ambulatory blood pressure monitoring. 
in the follow-up period. The eGFR values based on the Modification of Diet in Renal Disease (MDRD) Study Equation are shown in Table 3.

There were 82 patients that did not qualify for RDN procedure, all with a diagnosis of elevated BP despite optimal pharmacotherapy. The mean office BP measurements and mean ABPM scores for this group are shown in Table 4. For the patients who were disqualified from RDN, the reasons have been outlined in Table 5 .

Secondary hypertension due to various etiologies was diagnosed in 21 (25.6\%) of disqualified patients. The most frequent cause of secondary hypertension was a significant stenosis of at least 1 of the renal arteries (12 cases (14.6\%)). These patients were advised to continue with a diagnostic workup and treatment with vascular surgery. Seven patients $(8.5 \%)$ were found to have primary

Table 3. The eGFR values of the patients who underwent renal denervation calculated with the MDRD formula in $\mathrm{mL} / \mathrm{min} / 1.73 \mathrm{~m}^{2}$

\begin{tabular}{|l|c|c|c|}
\hline $\begin{array}{c}\text { Patient } \\
\text { No. }\end{array}$ & $\begin{array}{c}\text { eGFR } \\
\text { (baseline) }\end{array}$ & $\begin{array}{c}\text { eGFR } \\
\text { (at 6 months) }\end{array}$ & $\begin{array}{c}\text { eGFR } \\
\text { (at 12 months) }\end{array}$ \\
\hline 1 & 105.37 & 100.88 & 117.45 \\
\hline 2 & 85.01 & 89.29 & 87.81 \\
\hline 3 & 77.50 & 72.69 & 83.93 \\
\hline 4 & 85.64 & 91.10 & 87.75 \\
\hline 5 & 80.81 & 76.17 & 75.08 \\
\hline
\end{tabular}

eGFR - estimated glomerular filtration rate; eGFR 0, 6, 12 - values of estimated glomerular filtration rate during the first visit and during follow-up visits in the $6^{\text {th }}$ and $12^{\text {th }}$ month.

Table 4. Office BP measurements and ABPM measurements during the follow-up period in patients disqualified from RDN

\begin{tabular}{|l|c|}
\multicolumn{1}{|c|}{ Parameter } & Mean +SD \\
\hline \multicolumn{2}{|c|}{ Mean office BP measurements } \\
\hline Systolic BP & $159.20 \pm 8.88$ \\
\hline Diastolic BP & $92.20 \pm 6.54$ \\
\hline \multicolumn{2}{|c|}{ ABPM results } \\
\hline 24-hour systolic BP & $153.95 \pm 8.89$ \\
\hline 24-hour diastolic BP & $89.86 \pm 4.56$ \\
\hline Systolic BP - day & $158.45 \pm 8.89$ \\
\hline Diastolic BP - day & $91.44 \pm 6.15$ \\
\hline Systolic BP - night & $149.38 \pm 8.96$ \\
\hline Diastolic BP - night & $88.27 \pm 7.06$ \\
\hline
\end{tabular}

RDN - renal denervation; BP - blood pressure; SD - standard deviation; ABPM - ambulatory blood pressure monitoring.

Table 5. Main causes of disqualification from renal denervation during screening (number of patients)

\begin{tabular}{|l|}
\hline - Non-optimal pharmacotherapy (59) \\
- Secondary hypertension (21) \\
- significant stenosis in renal artery (12) \\
- primary hyperaldosteronism (7) \\
- Renal artery adrenal adenoma (2)
\end{tabular}

hyperaldosteronism and 2 (2.4\%) were discovered to have an active adrenal aldosterone-secreting adenoma and were referred to endocrinologists. Another 2 patients $(2.4 \%)$ had an abnormal renal artery diameters and kidneys with multiple vascular supplies.

Another 59 patients (72\%) were diagnosed with pseudo- $\mathrm{ResH}$, defined as an initial diagnosis of ResH but with suboptimal pharmacotherapy or patient noncompliance. At the end of the screening phase, optimization of therapy and counseling on compliance lead to this group achieving a satisfactory $\mathrm{BP}$ and disqualification from RDN. All the patients diagnosed with pseudo-ResH had initial BP measurements that could qualify them for RDN. The mean office BP and ABPM scores in the screening phase and after final effective pharmacotherapy administration are shown in Table 6.

In this pseudo-resistant group, a satisfactory BP was achieved at various points in the screening process. In all cases, pharmacotherapy was optimized during the initial screening visit and BP measurements were repeated at 6 weeks. The majority of patients (36) achieved satisfactory BP measurements at this point. In 23 of these cases, ResH was the working diagnosis until the first hospitalization. Despite some patients having BP measurements that qualified for RDN during the initial screening, not all of them were treated with diuretics. The pharmacotherapy regimen of the screening failure group on presentation and final pharmacotherapy regimen are shown in Fig. 3,4.

In most cases, the addition of hypertensive therapy was based on single-pill combinations with 2 or 3 substances. In 31 patients (52.5\%), we chose a triple combination pill of perindopril, amlodipine and indapamide. In 21 patients (35.6\%) a combination pill of perindopril and amlodipine was utilized.

In summary, an initial diagnosis of ResH was ruled out due to the discovery of reversible causes of high BP in 82 cases (94\%). Improper treatment or noncompliance issues were the cause of this in 59 of the patients (68\%).

Table 6. Office BP measurements and ABPM measurements including $\mathrm{BP}$ scores during the screening and final BP final scores after new pharmacotherapy optimization

\begin{tabular}{|l|c|c|c|}
\multicolumn{1}{|c|}{ Parameter } & $\begin{array}{c}\text { BP screening } \\
\text { scores } \\
\text { (mean } \pm \text { SD) }\end{array}$ & $\begin{array}{c}\text { BP final scores } \\
\text { (mean } \pm \text { SD) }\end{array}$ & p-value \\
\hline Systolic BP & $158.6 \pm 8.0$ & $125.30 \pm 5.81$ & $<0.001$ \\
\hline Diastolic BP & $93.4 \pm 5.4$ & $81.70 \pm 4.45$ & \\
\hline & ABPM results & & \\
\hline 24-hour systolic BP & $153.3 \pm 7.9$ & $123.9 \pm 1.9$ & \\
\hline 24-hour diastolic BP & $89.9 \pm 4.7$ & $74.4 \pm 1.9$ & \\
\hline Systolic BP - day & $157.8 \pm 7.9$ & $129.6 \pm 2.5$ & \\
\hline Diastolic BP - day & $91.6 \pm 6.5$ & $79.6 \pm 2.5$ & \\
\hline Systolic BP - night & $148.7 \pm 8.0$ & $118.3 \pm 3.3$ & \\
\hline Systolic BP - night & $88.2 \pm 6.9$ & $69.3 \pm 3.0$ & \\
\hline
\end{tabular}

BP - blood pressure; SD - standard deviation; ABPM - ambulatory blood pressure monitoring. 


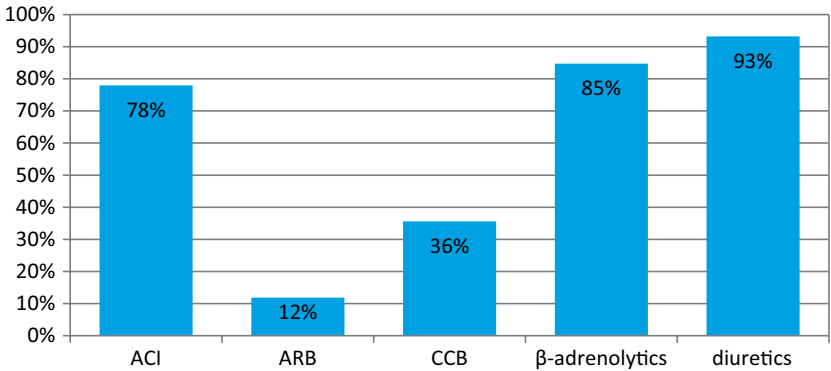

Fig. 3. Initial pharmacotherapy in patients with pseudoresistant hypertension

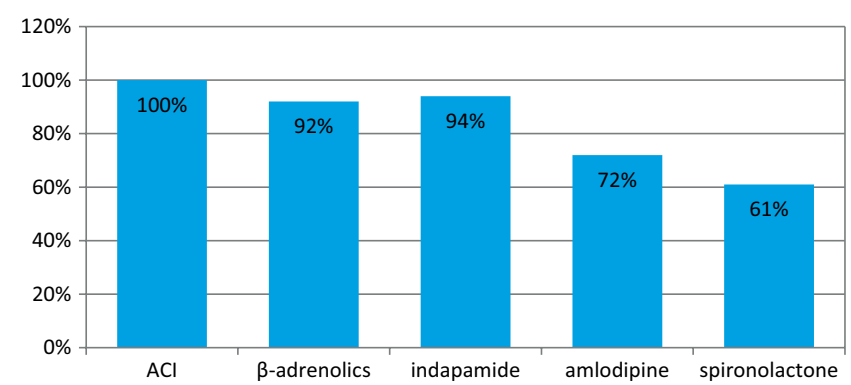

Fig. 4. Final pharmacotherapy in patients with pseudoresistant hypertension

\section{Discussion}

Resistant hypertension presents a clinical challenge often requiring an arduous diagnostic workup, pharmacovigilance, optimization of anti-hypertensive medications, and a detailed medical history to rule out non-compliance. Our study, to the best of our knowledge, is the first to analyze a population of patients initially presenting with a presumed diagnosis of $\mathrm{ResH}$ that in actuality suffered from uncontrolled hypertension caused by suboptimal pharmacotherapy or poor compliance issues.

Symplicity HTN-1 was the first clinical trial examining percutaneous transcatheter $\mathrm{RDN}$ in the treatment of ResH. ${ }^{4}$ The study consisted of a cohort of 50 patients who underwent RDN; a sustainable BP lowering effect was observed during consecutive follow-up visits at 1, 3, 6, 9, and 12 months. The cohort was then increased to 153 patients and the follow-up period was extended to 3 years. ${ }^{8}$ The hypotensive effect of RDN was still present 36 months from the date of the procedure. Blood pressure measurements were on average $32 \mathrm{~mm} \mathrm{Hg}$ lower for systolic BP and $14.4 \mathrm{~mm}$ $\mathrm{Hg}$ lower for diastolic BP as compared to the measurements at the initial screening. It was observed that the percentage of local femoral site complications was not any higher than those in similar procedures such as coronary angiography. Renal denervation did not have a negative impact on renal function. Other studies have also reported similar observations. ${ }^{9}$ Nevertheless, RDN is an invasive procedure and should only be performed in definitive cases of ResH.

There should be no ambiguity in the qualification of patients with ResH for RDN procedures. The results of our study show that ineffective medical therapy with 3 or more hypotensive drugs (including diuretics) is not enough to establish a definitive diagnosis. High BP in an outpatient setting may be a symptom of various disorders or of psychosomatic causes such as "white coat hypertension", which may be defined as persistently elevated BP $(\geq 140 / 90 \mathrm{~mm} \mathrm{Hg})$ "in the presence of a healthcare worker, particularly a physician" in patients not taking medication, with an average awake ABPM < 135/85 mm Hg. ${ }^{10}$ To obtain unadulterated objective measurements of BP, 24-hour ABPM is required. ${ }^{11}$ When suboptimal BP control is confirmed, it still requires exclusion of potentially reversible causes of HA. In our study $25.6 \%$ of the RDN disqualifications were caused by potentially reversible or secondary causes of hypertension, which generally have a prevalence of $5-10 \%$ in hypertensive patients. ${ }^{12}$ Renal diseases such as renal artery disease, glomerular and tubular diseases are responsible for almost $50 \%$ of cases of secondary hypertension. ${ }^{12}$ It is also important to note that diseases with significant deterioration of glomerular filtration excluded most of these patients from the RDN procedure in our study. Patients with serious renal artery stenosis need a more significant diagnostic workup due to stimulation of the renin-angiotensin-aldosterone system.

As demonstrated by our study and the literature, exclusion of secondary causes of HA is necessary. The most common and available methods to exclude this diagnosis are imaging techniques, most commonly renal Doppler ultrasonography. ${ }^{13}$ An RDN screening requires more specific methods that can in addition visualize the aorta and femoral arteries. Computed tomography (CT) with contrast is more appropriate, providing precise vascular imaging and providing visualization of the kidneys and adrenal glands to rule out any possible pathologies. Appreciation of the quantity and diameter of renal arteries is also necessary in preparing for RDN. In addition, renal and adrenal gland anomalies may be responsible for high BP which may be confirmed through imaging techniques.

Another significant cause of HA are pathologies resulting in the disruption of normal hormonal activity. ${ }^{14}$ In our study, we excluded 2 patients from RDN due to the presence of adrenal tumors discovered using CT. Detailed examination and diagnostic workup confirmed the hormonal, aldosterone-exerting activity of these tumors. Other patients with endocrine-disrupting properties had either primary hypertrophy of the renal cortex or primary hyperaldosteronism, diagnosed in 7 subjects in this group. Computed tomography was not useful in these cases, but, as the literature suggests, renal scintigraphy is more specific. ${ }^{15}$ Renal artery anatomy is another important issue that can influence the course, effectiveness and safety of the renal denervation procedure. Short, bendy and narrow vessels should be noted and may exclude patients from RDN. ${ }^{16}$ Furthermore, there is a group of patients with more than 1 artery supplying blood to the kidney. The general prevalence of this anomaly is seen in up to $28 \%{ }^{17}$ or $34 \%^{13}$ of patients with HA. In our study, we disqualified 2 patients from undergoing RDN due to the presence of multiple renal arteries in 1 kidney. 
The most common reason for disqualification from RDN in our study was inappropriate or suboptimal medical therapy which was identified in the initial screening phase of the study. In $72 \%$ of the cases, modifications of pharmacotherapy led to optimized BP control. A detailed medical history interview often revealed multiple therapeutic modifications leading to non-compliance. Numerous studies have been performed over the past decades discussing patient attitudes and compliance. It has been reported that satisfactory compliance is seen in only about $50 \%$ of cases. Partial compliance is seen in approx. $30-40 \%$ of patients, and $5-10 \%$ admit that they take their drugs selectively or do not take them at all. ${ }^{18}$ Achievement of optimal BP control by adding another drug as opposed to increasing the dose of the current regimen is a strategy confirmed by numerous studies. ${ }^{19}$ On the other hand, the effectiveness of therapy increases when the number of pills is reduced. ${ }^{20,21}$ The only way to reconcile these issues is single-pill combination therapy. ${ }^{19}$ In our study, patients disqualified from RDN during the initial screening phase due to potential drug compliance issues were ultimately transitioned to a single-pill triple combination (52.5\%) or single-pill double combination (35.6\%). In $28 \%$ of our cases, optimal BP control was achieved with the initiation of new pharmacotherapy, but only during the hospitalization period. These findings affirmed that drug compliance was the most important cause of false positive reports of ResH.

\section{Conclusions}

Renal artery denervation is an innovative method of treatment for $\mathrm{ResH}$ but remains debated, and its indication is decreasing. Improvements in technical skill and greater availability of devices for performing RDN are necessary. Additional studies must also be performed to further assess the benefits of the procedure.

The presence of uncontrolled high BP does not automatically warrant a diagnosis of $\mathrm{ResH}$, regardless of duration. Such cases always require thorough diagnostic testing to exclude secondary causes of hypertension. It is the up to the due diligence of healthcare workers to provide a conscientious choice of pharmacotherapy, taking into account the patient's capabilities and needs, to allow for good compliance.

Analyzing the methodology of our study, we hypothesize that the best way to achieve compliance is singlepill combinations of anti-hypertensives, but larger studies focused on the issues of compliance are required to verify this assumption.

\section{References}

1. Zdrojewski T, Rutkowski M, Bandosz P, et al. Prevalence and control of cardiovascular risk factors in Poland: Assumptions and objectives of the NATPOL 2011 Survey. Kardiol Pol. 2013;71(4):381-392.
2. Mancia G, Fagard R, Narkiewicz K, et al; Task Force Members. 2013 ESH/ESC Guidelines for the management of arterial hypertension: The Task Force for the management of arterial hypertension of the European Society of Hypertension (ESH) and of the European Society of Cardiology (ESC). J Hypertens. 2013;31(7):1281-1357.

3. Calhoun DA, Jones D, Textor S, et al. Resistant hypertension: diagnosis, evaluation, and treatment. A scientific statement from the American Heart Association Professional Education Committee of the Council for High Blood Pressure Research. Hypertension. 2008;51: 1403-1419.

4. Newcombe CP, Shucksmith HS, Suffern WS. Sympathectomy for hypertension. BrMed J. 1959;1(5115):142-144.

5. Krum H, Schlaich M, Whitbourn R, et al. Catheter-based renal sympathetic denervation for resistant hypertension: A multicentre safety and proof-of-principle cohort study. Lancet. 2009;373(9671):1275-1281.

6. Esler MD, Krum H, Sobotka PA, Schlaich MP, Schmieder RE, Böhm M; Symplicity HTN-2 Investigators. Renal sympathetic denervation in patients with treatment-resistant hypertension (The Symplicity HTN-2 Trial): A randomised controlled trial. Lancet. 2010;376(9756): 1903-1909.

7. Bhatt DL, Kandzari DE, O'Neill WW, et al; SYMPLICITY HTN-3 Investigators. A controlled trial of renal denervation for resistant hypertension. NEngl J Med. 2014;370(15):1393-1401.

8. Krum H, Schlaich MP, Sobotka PA, et al. Percutaneous renal denervation in patients with treatment-resistant hypertension: Final 3-year report of the Symplicity HTN-1 study. Lancet. 2014;383(9917):622-629.

9. Mahfoud F, Cremers B, Janker J, et al. Renal hemodynamics and renal function after catheter-based renal sympathetic denervation in patients with resistant hypertension. Hypertension. 2012;60(2): 419-424.

10. Pickering TG, Hall JE, Appel LJ, et al; Council on High Blood Pressure Research Professional and Public Education Subcommittee, American Heart Association. Recommendations for blood pressure measurement in humans: An AHA scientific statement from the Council on High Blood Pressure Research Professional and Public Education Subcommittee. J Clin Hypertens (Greenwich). 2005;7(2):102-109.

11. Verloop WL, VinkEE, Voskuil M, et al. Eligibility for percutaneous renal denervation: The importance of a systematic screening. J Hypertens. 2013;31(8):1662-1668.

12. Rimoldi SF, Scherrer U, Messerli FH Secondary arterial hypertension: When, who, and how to screen? Eur Heart J. 2014;35(19):1245-1254.

13. Hirsch AT, Haskal ZJ, Hertzer NR, et al. ACC/AHA Guidelines for the Management of Patients with Peripheral Arterial Disease (lower extremity, renal, mesenteric, and abdominal aortic): a collaborative report from the American Associations for Vascular Surgery/ Society for Vascular Surgery, Society for Cardiovascular Angiography and Interventions, Society for Vascular Medicine and Biology, Society of Interventional Radiology, and the ACC/AHA Task Force on Practice Guidelines (writing committee to develop guidelines for the management of patients with peripheral arterial disease)-summary of recommendations. J Vasc Interv Radiol. 2006;17:1383-1397.

14. Torre JJ, Bloomgarden ZT, Dickey RA, et al; AACE Hypertension Task Force. American Association of Clinical Endocrinologists Medical Guidelines for Clinical Practice for the diagnosis and treatment of hypertension. Endocr Pract. 2006;12(2):193-222.

15. Rubello D, Bui Ch, Casara D, Gross MD, Fig LM, Shapiro B. Functional scintigraphy of the adrenal gland. Eur JEndocrinol. 2002;147(1):13-28.

16. Verloop WL, Vink EE, Spiering W, et al. Renal denervation in multiple renal arteries. Eur J Clin Invest. 2014;44(8):728-735.

17. Gupta A, Gupta R, Singhla RK The accessory renal arteries: A comparative study in vertebrates with its clinical implications. J Clin Diagn Res. 2011;5(5):970-973.

18. Vermeire $E$, Hearnshaw $H$, Van Royen $P$, Denekens J. Patient adherence to treatment: Three decades of research. A comprehensive review. J Clin Pharm Ther. 2001;26(5):331-342.

19. Mancia G, Fagard R, Narkiewicz K, et al. 2013 ESH/ESC Guidelines for the management of arterial hypertension: The Task Force for the management of arterial hypertension of the European Society of Hypertension (ESH) and of the European Society of Cardiology (ESC). Eur Heart J. 2013;34(28):2159-2219.

20. Collier R. Reducing the "pill burden". CMAJ. 2012;184(2):E117-E118.

21. Bangalore S, Kamalakkannan G, Parkar S, Messerli FH. Fixed-dose combinations improve medication compliance: A meta-analysis. Am J Med. 2007;120(8):713-719. 